\title{
Desafíos de la pedagogía vocal en la música comercial contemporánea
}

Challenges of vocal pedagogy in contemporary commercial music

\author{
Camila $\operatorname{Arce}^{1}$ (1) (0) \\ ${ }^{1}$ Escuela Moderna de Música y Danza; Santiago de Chile; Chile.
}

Correspondencia

Camila Arce. E-mail:

perfeccionamientovoz@gmail.com

\section{Citar así}

Arce, Camila. (2021). Desafíos de la pedagogía vocal en la música comercial contemporánea. Revista de Investigación e Innovación en Ciencias de la Salud. 3(2),

119-127. https://doi.org/10.46634/riics. 75

Recibido: 08/07/2021

Revisado: 07/10/2021

Aceptado: 09/10/2021

\section{Invited editor}

Carlos Manzano Aquiahuatl, MD, MSc.

\section{Editor en jefe}

Jorge Mauricio Cuartas Arias, Ph.D. (ID)

\section{Coeditor}

Fraidy-Alonso Alzate-Pamplona, MSc.

Copyright $\mathbb{C}$ 2021. Fundación Universitaria María Cano. La Revista de Investigación e Innovación en Ciencias de la Salud proporciona acceso abierto a todo su contenido bajo los términos de la licencia Creative Commons Attribution-NonCommercial-NoDerivatives 4.0 International (CG BY-NG-ND 4.0).

\section{Declaración de intereses}

La autora ha declarado que no hay conflicto de intereses.

\section{Disponibilidad de datos}

Todos los datos relevantes se encuentran en el artículo. Para mayor información, comunicarse con el autor de

correspondencia.

\section{Financiamiento}

Ninguno. Esta investigación no recibió ninguna subvención específica de agencias de financiamiento en los sectores público, comercial o sin fines de lucro.

\section{Resumen}

Desde sus inicios, la pedagogía vocal de la música comercial contemporánea (término utilizado para referirse a la música popular, no lírica), se ha visto enfrentada a una serie de desafíos, dado a que inicialmente su canto fue enseñado de acuerdo con la técnica lírica, a pesar de contar con diferencias sustanciales en cuanto a la forma de emplear la voz, los recursos y registros vocales, propios de cada género musical, la amplificación externa, la formación y conocimientos necesarios para entrenar la voz, la evaluación y detección de problemas vocales, las herramientas pedagógicas necesarias para enseñar canto, entre otras distinciones. Lo anterior exige un replanteamiento de la pedagogía vocal y sus metodologías correspondientes para promover el aprendizaje de estudiantes de canto de música comercial contemporánea.

\section{Palabras clave}

Pedagogía vocal; voz; canto; música comercial contemporánea; pedagogía; entrenador vocal; cantante; desafíos; entrenamiento vocal; clase de canto; vocología.

\section{Abstract}

Contemporary commercial music vocal pedagogy has faced a number of challenges. This term is used to refer to popular or "non-lyrical" music. From its beginnings, the singing of contemporary commercial music was related and was taught based on lyrical technique, despite having substantial differences in the way of using the voice, such as the use of vocal registers, external amplification, and vocal resources of each musical genre, among other distinctions. Other challenges mentioned will be the training and knowledge necessary to train the voice, the evaluation and detection of vocal problems, and the pedagogical tools necessary to teach singing are challenges. Due to the aforementioned, there must be a rethinking of vocal pedagogy and its corresponding methodologies to promote the learning of contemporary commercial music singing students.

\section{Keywords}

Vocal pedagogy; voice; singing; contemporary commercial music; pedagogy; vocal coach; singer; challenges; voice training; voice lessons; vocology. 
Descargo de responsabilidad

El contenido de este artículo es responsabilidad exclusiva de la autora y no representa una opinión oficial de su institución ni de la Revista de Investigación e Innovación en Ciencias de la Salud.

\section{Agradecimientos}

Quiero agradecer a la Revista de Investigación e Innovación en Ciencias de la Salud y a sus editores, en especial al doctor Carlos Manzano, por la invitación a escribir este artículo.

\section{Introducción}

El concepto de música comercial contemporánea, abreviado como MCG, inicialmente fue propuesto por la maestra Jeannette LoVetri para reemplazar el término de música no clásica, una forma despectiva de referirse a los estilos de música popular [1]. Los estilos que abarca son variados y con características muy diferentes entre sí, algunos son el blues, el pop, el rock, el country, el soul, el teatro musical, entre otros [2].

Su masificación creó una demanda de lecciones para la interpretación de su canto por lo que profesores de canto lírico debieron adaptarse a la nueva generación de estudiantes que deseaban aprenderlo [3]. Así, y gracias a la rápida y fuerte tendencia, se creó la necesidad de elaborar una técnica pedagógica propia para la MCG; sin embargo, su desarrollo fue poco claro, basada exclusivamente en la experiencia del profesor y dependiente de lo que se enseñaba en la técnica lírica que, si bien es válida, no es necesariamente útil para cantar música comercial contemporánea.

Lo anterior impuso unos desafios que los profesores y estudiantes de la MCG deben enfrentar y resolver. Algunos de ellos son: 1) La tendencia a enseñar su canto con base en la técnica y tradición lírica; 2) La formación adicional que necesitan quienes desean impartir clases de canto; 3) Los conocimientos requeridos para la pedagogía vocal; 4) La manera de evaluar y detectar dificultades en la voz; 5) Las habilidades pedagógicas necesarias en un profesor o profesora de canto y 6) Las formas de favorecer el aprendizaje a largo plazo en la técnica vocal. Desafíos que este artículo tiene por objetivo analizar, desde distintas perspectivas y principalmente desde el habla hispana.

\section{Reflexión}

\section{Pedagogía enfocada en la técnica lírica}

Es común encontrar profesores que estructuran su metodología de enseñanza para el canto de la MCG en la técnica clásica, a pesar de que no son homologables ni en su manera de trabajar la voz ni en su estética musical [4].

En un comienzo, el canto lírico era interpretado por cantantes profesionales, en lugares que estaban debidamente diseñados para amplificar el sonido [5], por lo que no había necesidad de usar elementos externos, como micrófonos. En cambio, la música comercial contemporánea, cuyos orígenes se dan en los cantos de los esclavos africanos, denominados worksongs - quienes los entonaban en las faenas de plantación y recolección de algodón en Estados Unidos [6]-, no eran interpretaciones profesionales, pues los cantantes lo hacían más desde la tradición oral, el gusto y la intuición que desde un verdadero conocimiento musical. Esta forma de canto popular evolucionó a partir de la cualidad de speech-like, o habla coloquial [5], a lo que hoy conocemos como blues, género musical que se considera la base de casi todos los demás estilos de la MCG [7].

En 1920, el micrófono entra a la escena como una solución para aquellos cantantes de la MCG que daban conciertos y debían cantar más fuerte que los bronces y demás instrumentos que los acompañaban. A partir de ese momento el canto de la MCG es indivisible del uso de la amplificación y, por consiguiente, del uso del micrófono [1]. Lockheart nos indica que el micrófono trae consigo una nueva técnica vocal y la incorporación de recursos estilísticos que antes no eran posibles. Se les permite a las cantantes moverse en un rango más grave, utilizando voz mixta e incluso voz de 
pecho, también conocido como registro modal, poco utilizado en el canto lírico femenino [8]. Gracias al micrófono, el cantante de MCG ya no necesita utilizar la alta intensidad, que es un recurso utilizado por cantantes líricos como estrategia de amplificación, que le permiten usar recursos propios como la voz soplada, íntima y con mayor sutileza al disminuir la intensidad, volviendo a un canto más conversacional, percibido como más honesto y natural [9].

Como se viene sosteniendo, el canto lírico utiliza recursos vocales distintos a los del canto de la MCG. Entre estos recursos podemos destacar la ausencia de distorsiones vocales, el uso de la coloratura, las variaciones de extensión y la velocidad (extent y rate) del vibrato. Estas diferencias indican que el enfoque de la clase de canto de la MCG debe ser independiente del canto lírico, concentrada en su técnica, recursos y estilos propios.

Jeannette LoVetri menciona que "comparar la MCG [con la] clásica no sería lógico ya que el canto clásico no es útil como medida de producción vocal para los estilos de música comercial contemporánea" [5]. Si bien el instrumento vocal es el mismo para todos, los sistemas respiratorios, fonador y de resonancia se emplean de manera diferente al momento de cantar música comercial contemporánea. Esto lo podemos notar en la forma de emplear la musculatura laríngea, utilizar los registros vocales, el soporte respiratorio y los ajustes acústicos [3].

Para Robert Edwin decir "si aprendes a cantar lírico, puedes cantar cualquier cosa", es similar a que un instructor de tenis dijese: "Si aprendes a jugar tenis, podrás jugar cualquier deporte" [10]. Con esto enfatiza que un cantante que maneja la técnica lírica no necesariamente podrá desenvolverse en la técnica de música comercial contemporánea. Para esto, dicho cantante deberá entrenarse específicamente en canto de música comercial contemporánea.

Este es uno de los desafíos dentro de la pedagogía vocal de la MCG, debido a que un alumno de canto popular que aprenda la técnica de canto lírico podría retrasar su aprendizaje, ya que tendría que reeducar su voz y aprender nuevas formas de emitir sonido para lograr sonar como cantante de MCG.

\section{Formación para la enseñanza del canto de la MCC}

Existen varios dilemas entre quienes dictan clases de canto para saber quién está realmente capacitado para ser un buen profesor. Ante esto cabe preguntarse si un cantante profesional, un logopeda, un fonoaudiólogo o un licenciado en música, es un buen profesor de canto por saber utilizar su propia voz o conocer teóricamente el instrumento.

Brian Gill y Christian Herbst indican que no se puede asumir que una persona que ha trabajado como cantante toda su vida haya adquirido suficiente conocimiento objetivo sobre anatomía, fisiología y acústica como para impartir lecciones de canto [11]. Se recalca la palabra "objetivo", pues un gran desafío de la pedagogía vocal es lo que Claudia Mauleón llama "terminología personal" [12], que usa términos subjetivos propios del profesor que los enseña, basados en sus propias sensaciones y percepciones.

Suele suceder que quien sabe cantar o quien canta bello enseña el arte del canto [13], pero

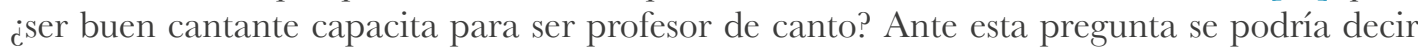
que a pesar de que un cantante conozca su voz y sepa utilizarla de manera saludable y eficaz, pudiéndola adecuar al repertorio, no necesariamente cuenta con suficientes herramientas pedagógicas ni todo el conocimiento necesario para impartir lecciones de canto de manera profesional. La enseñanza de técnica vocal debe adecuarse a las necesidades fisiológicas del alumno como individuo para así sacar provecho a su instrumento [11] y viceversa. 
Según una investigación realizada por Irene Bartlett y Marisa Naismith [3], nueve profesores de canto, reconocidos a nivel internacional, indicaron durante una entrevista que tuvieron que formarse de manera empírica al no haber una preparación profesional estructurada para desempeñarse como cantantes de MCG. Por otra parte, no existe una fiscalización de la labor de los maestros de canto sin estudios formales que garantice sus capacidades pedagógicas [3].

\section{Conocimientos necesarios para la enseñanza del canto de la MCC}

A grandes rasgos, un profesor o profesora de canto debe desenvolverse en los siguientes elementos musicales; 1) rítmicos, 2) melódicos y 3) armónicos, así como saber relacionarlos y separarlos cuando es preciso, además de complementarlos con la emotividad y expresión necesaria para interpretar una pieza musical y transmitir el mensaje de lo que se está cantando. Deben manejar sus diversos géneros, lo que implica tener conocimientos específicos, ya que cada estilo requiere de habilidades y cualidades sonoras distintas y el mercado musical exige adaptabilidad a los intérpretes de canto [14]. Adicionalmente, un docente de canto debiese tener experiencia como intérprete profesional [4]; es decir, desempeñarse como cantante en conciertos en vivo o como músico sesionista, por citar algunos campos.

Brian Gill y Christian Herbst [11] concluyeron que el entrenamiento vocal no solo requiere cantar con gran experticia, sino que también es necesario contar con conocimientos profundos en anatomía, fisiología y acústica de la producción vocal. Daniel Marconi et al mencionan que tanto cantantes profesionales como aficionados no cuentan con estudios en anatomía, fisiología y patologías de la voz cantada [15], siendo que estos conocimientos son de gran importancia para desarrollar una técnica sana y prevenir lesiones vocales dentro de las salas de clases. Además, un profesor de canto debe saber explicar e implementar esta información dentro de un escenario pedagógico sin agobiar al estudiante.

Una de las importancias de la comprensión de la anatomía y fisiología de la voz es que esto implica trabajar la voz de manera integral. La tendencia de los maestros de canto, al entrenar a estudiantes sin conocer adecuadamente la anatomía y la fisiología de la voz, es trabajar el instrumento aislando los tres subsistemas y secuenciando la voz; es decir, primero respiración, después fonación y luego resonancia. Sin embargo, la teoría "no-lineal fuente filtro" definida por el Dr. Ingo Titze, estipula que existe una relación de retroalimentación entre los tres subsistemas de producción de la voz, a saber: 1) sistema respiratorio 2) sistema fonatorio 3) sistema resonancial. Los cuales, al interactuar entre sí, son inseparables dado que trabajan en conjunto para la producción de la voz $[16,17]$. En palabras sencillas, esto significa que los cambios en el sistema respiratorio afectan al sistema fonador y resonancial como también los cambios en el sistema resonancial afectan el sistema respiratorio y fonador, lo que implica que el entrenamiento del canto debe efectuarse trabajando el instrumento de manera conjunta, sin aislar los subsistemas ya que de no ser así trabajaríamos la voz fuera del contexto de producción vocal y este entrenamiento no sería transferible al canto mismo.

Matthew Edwards \& Matthew Hoch indican que "la pedagogía basada en hechos permite a los profesores establecer las dificultades técnicas y proveer correcciones apropiadas" [9]. Por ello es importante que los profesores de canto complementen su experiencia con las investigaciones científicas que buscan explicar y dar sustento a las prácticas tradicionales dentro de las clases. Actualizar continuamente la información a través de los avances de la ciencia vocal permite que los profesores de canto fundamenten sus metodologías en elementos objetivos y no en suposiciones ni "lenguaje mitológico" [18]. Claudia Mauleón plantea una "revisión y renovación de las prácticas tradicionales con base en los descubrimientos de la ciencia vocal" [12]. 
Cabe señalar que a través de la ciencia vocal es posible llegar a ciertos consensos en cuanto a los conceptos y definiciones dentro del vocabulario de técnica y recursos vocales, para homogeneizar el lenguaje y la comunicación entre profesionales de la voz, ya sean fonoaudiólogos, locutores, profesores de canto, cantantes u otros.

Un ejemplo de esto es el concepto de registro vocal, definido en 1894 por Manuel García como "una serie de sonidos homogéneos consecutivos producidos por un mecanismo que difieren esencialmente de otra serie de sonidos igualmente homogéneos producidos por un mecanismo diferente" $[19,20]$. Sin embargo, hasta el día de hoy se ve cómo este concepto es comúnmente confundido para definir la tesitura: "tonos del más grave al más agudo que una persona puede emitir con comodidad y estética" [21] o clasificación vocal de un cantante (dígase soprano, alto, tenor) e incluso se combinan los registros vocales a sensaciones de vibración, particularmente en el pecho o cabeza y no mecanismos laríngeos. También, es importante entender que con el pasar los años los conceptos van mutando, adaptándose, lo que exige a los profesores de canto mantenerse en constante actualización.

\section{Evaluar y detectar dificultades en la voz}

La labor de un profesor de canto se basa en educar y entrenar la voz de sus estudiantes mediante ejercicios y aplicación de la técnica al repertorio para que "el estudiante pueda cantar cómodamente sonidos de duraciones, cualidades y colores específicos" [22], entre otros aspectos a considerar. Un profesor de canto debe contar con oído entrenado para poder detectar la causa del problema vocal, como de una voz entrenada, para demostrar correctamente el sonido o técnica que requiere el alumno y poder corregir adecuadamente.

Un factor de suma relevancia dentro de una clase de canto es determinar las necesidades del alumno para que éste pueda lograr el sonido con alta economía vocal, también definido como el máximo output acústico con la menor cantidad de estrés de impacto o carga biomecánica [20]. Para esto, el profesor de canto debe saber evaluar y establecer lo que hay que entrenar en la voz a través del input que escucha, lo que percibe visualmente y de la retroalimentación de sensaciones del estudiante.

La capacidad de analizar la voz auditivamente en términos objetivos (función, producción, etc), independiente de los gustos individuales del oyente, es necesaria para diagnosticar las deficiencias técnicas o identificar si hay alguna patología vocal u otro problema de salud, según metodologías de pedagogía vocal, tales como, Complete Vocal Technique ${ }^{\mathrm{TM}}$, Somatic Voicework $^{\mathrm{TM}}$, New York Vocal Coaching ${ }^{\circledR}$, Modern Vocal Training, entre otras. Esto implica que el profesor de canto debe saber evaluar de manera objetiva el estado de la voz, si ésta es saludable, si los registros vocales están balanceados, la cualidad y otros elementos vocales. Así el profesor recopila síntomas para establecer la causa o causas del desbalance que percibe para así proveer correcciones certeras. El maestro debe transmitir esta habilidad a su alumno para que éste también pueda aprender a evaluar su propia producción vocal.

\section{Habilidades de los profesores de canto}

Por otro lado, el profesor de canto debería ser capaz de cantar y así demostrar los ejercicios vocales que les exige a sus estudiantes. Estos ejercicios deben tener una finalidad que vaya de la mano con lo que el entrenador desea trabajar en el estudiante. Cada ejercicio actúa como un estímulo que busca una respuesta en cuanto a mecanismo vocal, cualidad sonora o interpretación. El profesor debe escoger las variables del ejercicio: la tarea fonatoria, velocidad, intensidad, fonética, color, registro vocal, entre otras variables, de acuerdo con la respuesta 
vocal que espera del alumno. Ningún ejercicio se da al azar ya que estos van directamente vinculados con la respuesta fisiológica o la cualidad de sonido que se busca. Como todo cuerpo es distinto, el mismo ejercicio puede resultar en respuestas distintas, dependiendo del individuo. Es por esto que un maestro de canto debe conocer las variables y comprender las reacciones del estudiante para así modificar o mantener las variables para un mejor resultado.

Un ejemplo de esto es el uso de la velocidad en un ejercicio. Si un estudiante tiene problemas con el patrón melódico y no puede mantener la afinación cuando canta la melodía establecida, lo más probable es que el profesor baje la velocidad del ejercicio, incluso puede seccionar el ejercicio hasta que el alumno comprenda las notas y luego incrementará la velocidad en la medida que el alumno logre mantener la afinación. Si el maestro decidiese continuar a la misma velocidad, el ejercicio ya no sería útil y lo más probable es que produzca frustración en el alumno quien no podrá cantar la melodía de manera precisa. Esto consiste en una variable, un problema y un tipo de solución, cuya respuesta no significa que sea siempre la misma para los distintos individuos.

Como conclusión, podemos afirmar que en un entrenador vocal, las capacidades de observación e identificación de la causa de problemas y necesidades puntuales del alumno son indispensables. Por este motivo, un maestro de canto no sólo debe tener su voz entrenada sino que debe contar con las habilidades auditivas para poder escuchar, diagnosticar y solucionar los desafios que encuentra el alumno al momento de cantar.

\section{Aprendizaje a largo plazo}

El aprendizaje del canto, de manera profesional, incluye el estudio de técnica vocal, teoría y armonía musical, géneros musicales, puesta en escena como también danza, grabación en estudio, entre otros aspectos. Sin embargo, dentro de la carrera de un cantante profesional no se requiere contar con herramientas pedagógicas para desempeñarse como artista, por lo que un cantante profesional con estudios de canto o música no necesariamente ha aprendido los recursos necesarios para enseñar.

Se ha visto que los aprendizajes más significativos son cuando el profesor guía al estudiante mediante el uso de sensaciones, imitación, motivación intrínseca para generar un impacto a largo plazo en el proceso de aprendizaje. En la pedagogía vocal es común que sean utilizadas instrucciones biomecánicas, tales como; abrir la boca, contraer el abdomen, levantar el velo del paladar, entre otras. No obstante, este tipo de instrucciones suelen ser muy complejas y solo favorecen una buena ejecución en ese determinado momento; es decir, favorecen un aprendizaje a corto plazo.

Por otro lado, enseñar canto utilizando el aprendizaje sensoriomotor es una manera de propiciar el desarrollo del control motor a través de procesos prácticos y demostraciones, lo que favorece el desarrollo de movimientos físicos coordinados, tal como el uso de la voz. [23] Este tipo de aprendizaje involucra la memoria procedural y por ende favorece la permanencia de lo aprendido en el tiempo. Manejar e incorporar estos principios en las lecciones de canto significa que "el aprendizaje será de largo plazo, estable y con cambios relativamente permanentes" $[16,24]$. Utilizar los principios del aprendizaje sensoriomotor implica que la atención del estudiante será externa y no estará enfocada en la biomecánica [25].

Para abordar este tipo de aprendizaje es necesario que el profesor reconozca las particularidades de la voz de su alumno y cómo facilitar y adecuar la clase para lograr mayor comprensión y retención de lo enseñado [26]. 
Un aspecto adicional que debe ser considerado al momento de enseñar canto es el aspecto psicosocial del estudiante. La empatía y el respeto son fundamentales para crear un ambiente seguro que favorezca el aprendizaje. Todo profesor tiene el poder de motivar y apoyar a un alumno o desalentarlo, ya sea a través de su método de enseñanza o la manera en que se comunica con él. Hay que tomar en consideración el proceso del alumno, ser flexible y atento no solo a los aspectos técnicos/musicales, sino que también a la persona, su estado de ánimo y evolución durante la clase.

\section{Conclusión}

Los profesionales de la voz y profesores de canto deben tomar conciencia de estos desafíos para así enfrentar las dificultades que se presentan durante sus clases. Es necesario que quienes trabajan en el área de la voz cuestionen e investiguen la información de las prácticas tradicionales provenientes del canto lírico y evalúen de dónde y con qué fundamentos fue implementada dicha información para enseñar técnica vocal y si esta se aplica al canto de la MCC. Además, el profesor o profesora de canto debe complementar su experiencia con conocimientos científicos y continuar avanzando hacia prácticas más sanas y concretas, las cuales seguro promoverán el progreso de los alumnos.

Por ello es importante una reestructuración de las metodologías del canto de música comercial contemporánea, independiente de la técnica lírica y a cargo de profesionales capacitados específicamente en pedagogía vocal, que incorporen herramientas pedagógicas en sus salas de clases.

\section{Referencias}

1. Weekly EM, Lovetri JL. Follow-up Contemporary Commercial Music (CCM) Survey: who's teaching what in nonclassical music. J Voice. 2009 May;23(3):367-375. Doi: https://doi.org/10.1016/j.jvoice.2007.10.012

2. American Academy Of Teachers Of Singing. In Support of Contemporary Commercial Music (Nonclassical) Voice Pedagogy. Journal of Singing. 2008;65(1):7-10. Available: https://n9.cl/agwbd

3. Bartlett I, Naismith ML. An Investigation of Contemporary Commercial Music Voice Pedagogy: A class of its own? Journal of Singing. 2020;76(3):273-280. Available: https:// n9.cl/il7k8

4. Rooney T. The Understanding of Contemporary Vocal Pedagogy and the Teaching Methods of Internationally Acclaimed Vocal Coaches. International Journal of Learning, Teaching and Educational Research. 2016;15(10):147-162. Available: https://www.ijlter. org/index.php/ijlter/article/view/762

5. LoVetri J. Contemporary Commercial Music. J Voice. 2008;22(3):260-262. Doi: https:// doi.org/10.1016/j.jvoice.2006.11.002

6. Ward GC, \& Burns K. Jazz: A History of America’s Music.1st ed. New York: Knopf; 2000.

7. Keskinen AK. Contemporary Commercial Music Education and Different Cultural Contexts. Vocal Pedagogy and Contemporary Commercial Music: Reflections on Higher Education Non-Classical Vocal Pedagogy in the United States and Finland. Published. 2013. Available: http://urn.fi/URN:NBN:fi-fe201306103898 
8. Lockheart P. A History of Early Microphone Singing, 1925-1939: American Mainstream Popular Singing at the Advent of Electronic Microphone Amplification. Popular Music and Society. 2003;26(3):367-385. Doi: https://doi.org/10.1080/0300776032000117003

9. Edwards M, Hoch M. CGM versus Music Theater: A Comparison. Journal of Singing, Volume. 2018;75(2):183-190. Available: https://n9.cl/zs8lm

10. Edwin R. From Classical to Pop: A Case Study. Journal of Singing. 2000;56(3):71-72. Available: https://n9.cl/9rir8

11. Gill BP, Herbst CT. Voice pedagogy — what do we need? Logopedics Phoniatrics Vocology. 2015;41(4):168-173. Doi: https://doi.org/10.3109/14015439.2015.1079234

12. Mauléon-Stäheli C. Arte y ciencia. Hacer y pensar la Pedagogía Vocal. Revista De Investigaciones En Técnica Vocal. 2015;1:78-87. Available: https://revistas.unlp.edu.ar/ $\mathrm{RITeV} /$ article/view/2061

13. Stark J. Bel Canto: A History of Vocal Pedagogy. 2nd ed. University of Toronto Press: Scholarly Publishing Division;2003.

14. LoVetri J. The Necessity of Using Functional Training in the Independent Studio. Journal of Singing. 2013;70(1):79-86. Available: https://n9.cl/38ann

15. Rodríguez Marconi D, Morales Cárdenas C, Gaete Antilen L, Garrido Ormeño M, Pardo Reyes C. Level of physiological, anatomical and pathological knowledge of the singing voice on the part of professional and amateur singers. Revista CEFAC. 2018;20(5):621631. Doi: https://doi.org/10.1590/1982-021620182054118

16. Titze I, Riede T, Popolo P. Nonlinear source-filter coupling in phonation: Vocal exercises. The Journal of the Acoustical Society of America. 2008;123(4):1902-1915. Doi: https://doi.org/10.1121/1.2832339

17. Garcia M. Hints on Singing Translated from the French by Beata Garcia New \& Revised. 2nd ed. E. Ascherberg \& Co;1894.

18. Alessandroni N. Pedagogía vocal contemporánea y profesionales prospectivos: hacia un modelo de diagnóstico en técnica vocal. Boletín de Arte. 2012b;13(13):72-76. Available: http://papelcosido.fba.unlp.edu.ar/ojs/index.php/boa/article/view/216

19. Henrich N. Mirroring the voice from Garcia to the present day: Some insights into singing voice registers. Logopedics Phoniatrics Vocology. 2006;31(1):3-14. Doi: https://doi. org/10.1080/14015430500344844

20. Calvache C, Guzman M, Bobadilla M, Bortnem C. Variation on Vocal Economy After Different Semi Occluded Vocal Tract Exercises in Subjects With Normal Voice and Dysphonia. Journal of Voice. 2020;34(4):582-589. Doi: https://doi.org/10.1016/j.jvoice.2019.01.007

21. Jackson-Menaldi CA. La voz normal. 1 ed. Madrid: Panamericana; 2005.

22. Sergius Kagen. On Studying Singing. 1 ed. New York: Dover Publications Inc; 2009. 
Desafíos de la pedagogía vocal

Arce

23. Acevedo K, Guzmán M. Efectos del entrenamiento respiratorio aislado en las variables respiratorias y vocales. Revista de Investigación e Innovación en Ciencias de la Salud. 2020;2(2):56-69. Doi: https://doi.org/10.46634/riics.51

24. Titze IR, Abbott KV. Vocology: The Science and Practice of Voice Habilitation. National Center for Voice and Speech. Compton Publishing Ltd; 2021

25. Bergan C. Motor Learning Principles and Voice Pedagogy: Theory and Practice. Journal of Singing. 2010;66(4):457-468.

26. Guzmán M. Principios de Aprendizaje Sensorio - Motor. Apuntes de Aprendizaje Módulo 8 Vocal Coach. 2019. Available: https://n9.cl/uyn0q 